\title{
The Treatment of Infantile Cafe Au Lait Spot Using Dr. Hoon Hur's Golden Parameter Therapy with a High Fluence 1064nm Q-Switched Nd: Yag Laser
}

\author{
Hoon Hur*, Duck Taik Shim, Sung Eun Song, Pyoung Su Kim, Dong Nyeok Hyun and Yu Ri Kim \\ Choice Dermatology Clinic, Pyeongchon, Korea
}

Received: March 18, 2020; Accepted: March 24, 2020; Published: March 26, 2020

*Corresponding author: Hoon Hur, Choice Dermatology Clinic, 1045, Hogye-dong, Dongan-gu, Anyang-si, Gyeonggi-do, Korea. E-mail: dermahur@naver.com

\begin{abstract}
Background: Infantile Café-Au-Lait Spot (CALS) is a benign hyperpigmentary skin disorder that ranges in color from light brown to dark brown. Using traditional laser therapies including 532nm potassium titanyl phosphate(KTP) laser, 694nm ruby laser and $755 \mathrm{~nm}$ alexandrite laser for infantile CALS treatment may provoke harmful side effects such as purpurae, crusts,Post-Inflammatory Hyperpigmentation $(\mathrm{PIH})$, mottled hypopigmentation and scarring. Unfortunately, there is no clear standard for the laser treatment of infantile CALS because an infant is too young to receive laser treatment.

Objectives: This study was performed to investigate the efficacy and safety of Dr. Hoon Hur's Golden Parameter Therapy (GPT) with a high fluence $1064 \mathrm{~nm}$ Q-switched Nd:YAG laser laser (QSNL) for infantile CALS treatment.
\end{abstract}

Methods: Nineteen Korean patients with infantile CALS were enrolled in this study and treated with a $1064 \mathrm{~nm}$ QSNL on a weekly basis for 20-50 treatment sessions of Dr. Hoon Hur's GPT. The parameters were a spot size of $7 \mathrm{~mm}$, a fluence of $2.2 \mathrm{~J} / \mathrm{cm} 2$ and a pulse rate of $10 \mathrm{~Hz}$ with one pass by a sliding-stacking technique over the infantile CALS.

Results: After the final treatment, all of the 19 patients with infantile CALS were achieved the complete removal of pigmented lesions without any side effects such as purpurae, crusts, $\mathrm{PIH}$, mottled hypopigmentation or scarring. No recurrences were observed in any of the patients after a follow- up of 6-14 months.

Conclusion: We suggest that Dr. Hoon Hur's GPT with a high fluence $1064 \mathrm{~nm}$ QSNL is a safe and effective treatment for infantile CALS without causing side effects and recurrences.

Keywords: Infantile Cafe au Lait Spot; 1064nm Nd:YAG laser; Dr Hoon Hur's GPT

\section{Introduction}

Café Au Lait Spot (CALS), a benign cutaneous pigmentary disorder with light or dark brown coloration, may occur on any parts of the body excluding palms and soles at birth or in infancy. The size may range from $0.5 \mathrm{~cm}$ to $30 \mathrm{~cm}$ in diameter. The histopathologic findings show that there is no nevus cell in the basal layer of epidermis and that CALS does not develop into malignant lesion [1-3]. Treatment for CALS is not necessary except for cosmetic purposes [1-3]. Especially, infantile CALS is very hard to treat with out causing any side effects and recurrences because an infant is too young to receive laser treatment [4-6]. Therefore, this study was undertaken to confirm the efficacy and safety of Dr. Hoon Hur's Golden Parameter Therapy (GPT) using a high fluence 1064nm Q-switched Nd:YAG laser (QSNL) for treating infantile CALS without any side effects and recurrences.

\section{Materials and Methods}

A total of nineteen Korean patients (age range: 3-11months old, mean age: 6.3 months old) who were clinically diagnosed with infantile CALS (Figures1,3,6) participated in this study and they had no significant medical or familial history. Written consents were received from all of the parents of patients before proceeding into treatment. Prior to the laser treatment, 9.6\% lidocaine cream of topical anesthetics (Inistbio, Hawsung, South Korea) was applied on the infantile CAL Slesion of patients for 20 minutes under occlusion.Then,the patients were underwent 2050 treatment sessions of Dr. Hoon Hur's GPT using a high fluence $1064 \mathrm{~nm}$ QSNL (StarWalker Laser, Fotona, Slovenia) on a weekly basis with a spot size of $7 \mathrm{~mm}$, a fluence of $2.2 \mathrm{~J} / \mathrm{cm}^{2}$ and a pulse rate of $10 \mathrm{~Hz}$ with one pass by a sliding-stacking technique over the infantile CALS. After each laser treatment, the lesion of infantile CALS was cooled with ice packs but the patients did not use a broad-spectrum sunscreen. To evaluate the result, standardized digital photography with a Canon Camera G11 (Japan) was used by comparing photos taken on the first day of the treatment to those taken 4 weeks after the final treatment session. The physician's clinical assessment of the degree of improvement of the patients (mean score of two investigators who did not attend the treatment) was also carried out 4 weeks after the last treatment session and reported as percentage resolution as follows: poor ( $0-25 \%$ clearance), fair $(26-50 \%$ clearance), good (51-75\% clearance), excellent (76-95\% clearance)and complete (96-100\% clearance) by analyzing the clinical photographs of patients. The parents of patients were asked to report any side effects, pain or discomfort during the treatment. 


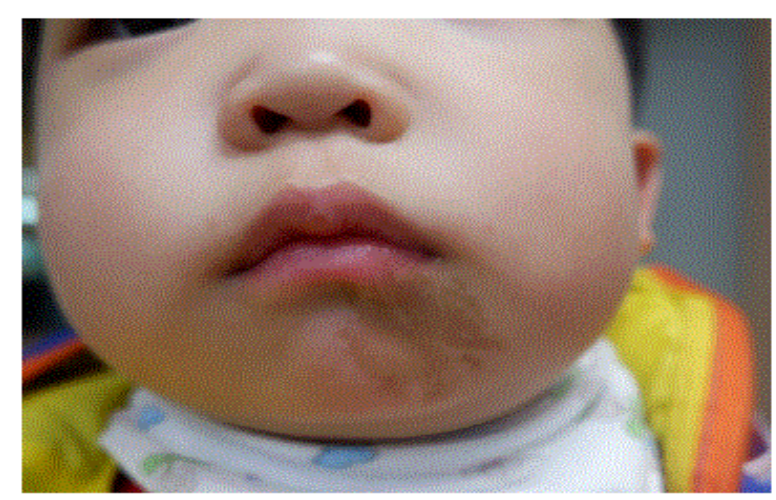

Figure 1: Infantile CALS on the Lower Jaw (Before Treatment)

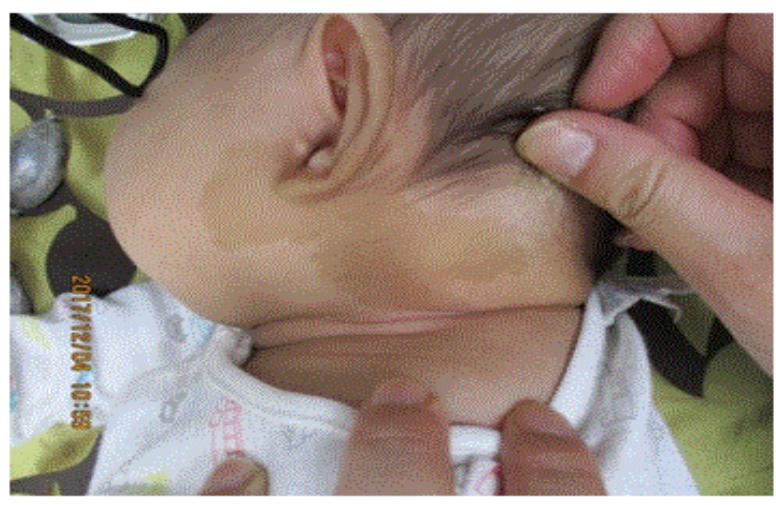

Figure 3: Infantile CALS on the left side of face and neck (before treatment:12/4/2017)

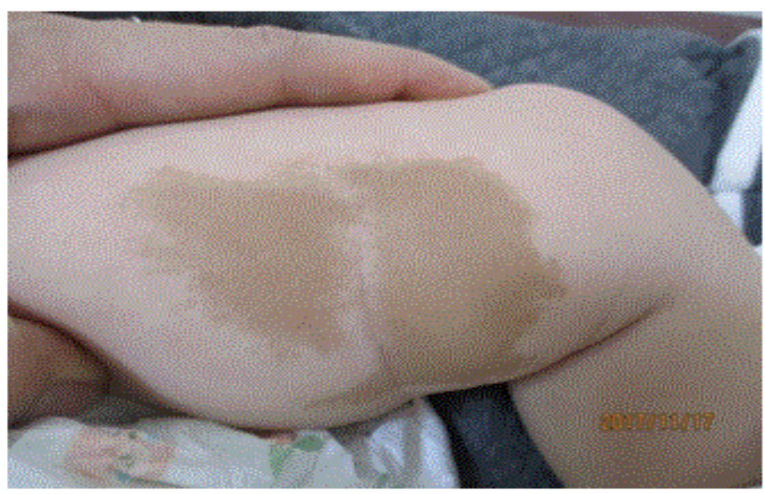

Figure 6: Infantile CALS on the left inner thigh (before treatment:11/17/2017)

\section{Results}

Nineteen Korean patients with infantile CALS were involved in this study (Table 1). All of the 19 patients with infantile CALS were achieved complete clearance of the pigmented lesions (Table 2). There was no side effects reported including purpurae, crusts, PIH, mottled hypopigmentation or scarring except slight
Table 1. The demographic data of 19 patients with infantile CALS and the characteristics of infantile CALS

\begin{tabular}{lr}
\hline \hline Age & \\
Age range & $3-11$ months old \\
Mean age & 6.3 months old \\
\hline Gender & \\
Male & $6 / 19(40 \%)$ \\
Female & $9 / 19(60 \%)$ \\
\hline Family history & $(-) ;$ unremarkable \\
\hline Location & \\
Face & $7 / 19(36.8 \%)$ \\
Trunk & $4 / 19(21.1 \%)$ \\
Arm & $3 / 19(15.8 \%)$ \\
Thigh & $2 / 19(10.5 \%)$ \\
Lower leg & $3 / 19(15.8 \%)$ \\
\hline Characteristics of lesion & \\
Bright brown color & $7 / 19(36.8 \%)$ \\
Dark brown color & $12 / 19(63.2 \%)$ \\
Homogenous round shape & $6 / 19(31.6 \%)$ \\
Heterogenous irregular shape & $13 / 19(68.4 \%)$ \\
\hline \hline
\end{tabular}

\begin{tabular}{lc} 
Table 2. The result of treatment with Dr. Hoon Hur's GPT \\
\hline \hline Treatment response & Number of patients \\
\hline Poor (0-25\% clearance) & 0 \\
Fair ( $26-50 \%$ clearance) & 0 \\
Good ( $51-75 \%$ clearance) & 0 \\
Excellent (76-95\% clearance) & 0 \\
Complete ( $96-100 \%$ clearance) & $19 / 19(100 \%)$ \\
\hline \hline
\end{tabular}

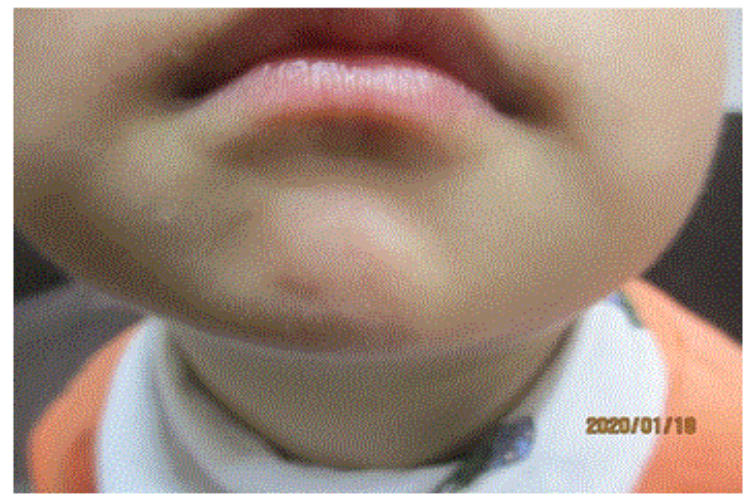

Figure 2: A complete clearance of infantile CALS (after Dr. HoonHur's GPT)

pain during the laser treatment (Figures 2,4,7).No recurrences have been found after a follow-up of 6-14months (Figures 5,8)

Citation: Hoon Hur, Duck Taik Shim, Sung Eun Song, et al (2020) The Treatment of Infantile Cafe Au Lait Spot Using Dr. Hoon Hur's Golden Parameter Therapy with a High Fluence 1064nm Q-Switched Nd: Yag Laser. Clin Res Dermatol Open Access 7(1): 1-5. DOI: 


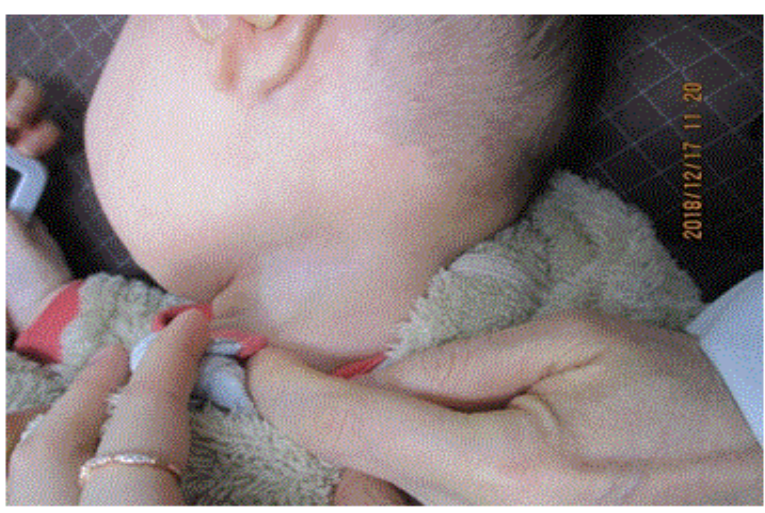

Figure 4: A complete clearance of infantile CALS (after Dr. HoonHur's GPT:12/17/2018)

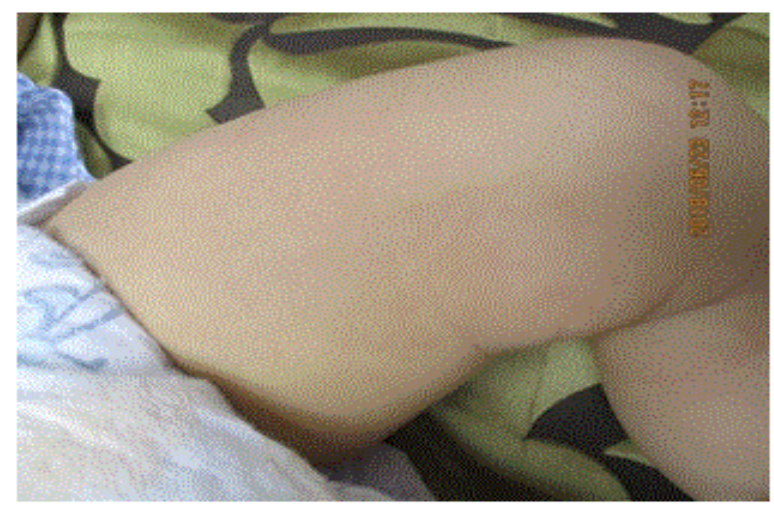

Figure 7: A Complete clearance of infantile CALS (after Dr. HoonHur's GPT:6/23/2018)

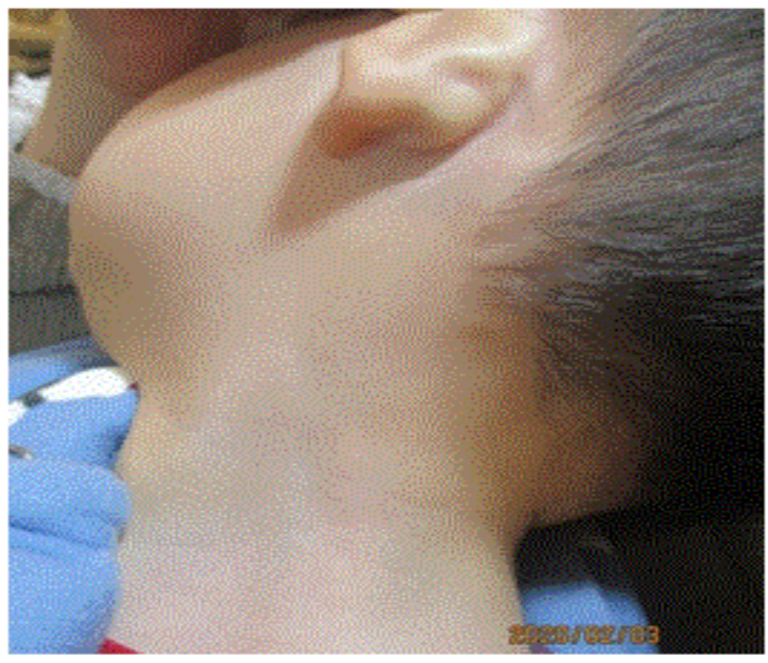

Figure 5: There is no recurrence at 14 months' follow-up (2/3/2020).

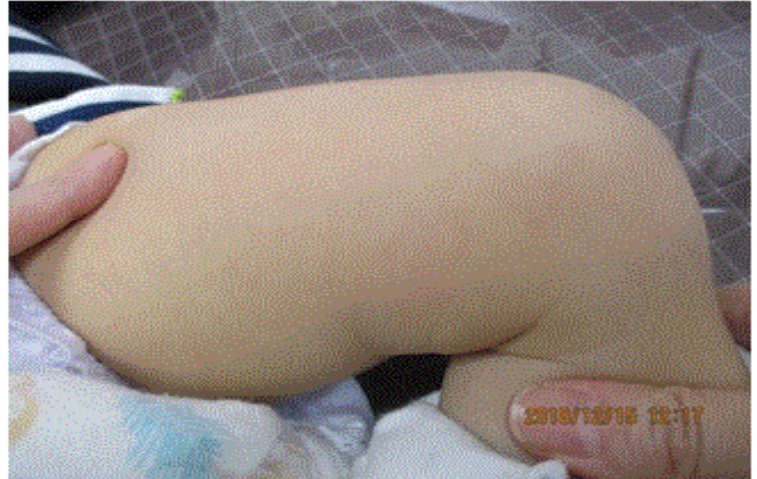

Figure 8: There is no recurrence at 6 months' follow-up (12/15/2018).

\section{Discussion}

Café Au Lait Spot (CALS) is a benign cutaneous pigmentary disorder, presenting as a hyperpigmented patch with a sharp border [1-3]. CALS can be classified into two different types. The common one is the non-syndromic solitary CALS and the other one is more rare and associated with genetic syndrome which includes neurofibromatosis type 1, McCune-Albright syndrome and tuberous sclerosis, called the multiple Café Au Lait Spots (CALSs) [1,7]. In terms of non-syndromic solitary CALS, NF1 somatic mutations do not occur neither in the melanocytes and keratinocytes, nor in the surrounding normal skin, and the epidermal melanocytes do not contain macromelanosomes [1,7]. On the other hand, regarding multiple CALSs with neurofibromatosis type1, NF1 somatic mutations can occur in the melanocytes and keratinocytes in the epidermis and fibroblasts in the dermis. In addition, NF1 somatic mutations can be found in the surrounding normal skin, and the epidermal melanocytes include macromelanosomes [1,7]. In non-syndromic solitary CALS, the expression of endothelin- 1 is increased in the keratinocytes and the both expression of Stem Cell Factor (SCF) and Hepatocyte Growth Factor (HGF) are increased in the fibroblasts compared to those of normal skin. These increased expressions of endothelin-1, SCF and HGF, which lead to the activation of the melanocytes, enhance melanin synthesis in the melanosomes, eventually causing CALS [8-10]. Especially, both multiple CALSs with neurofibromatosis type 1 and normal skin with neurofibromatosis type 1 show higher increase of expression of the endothelin-1 in the keratinocytes, and higher increase of expression of the SCF and HGF in the fibroblasts compared to those in the non-syndromic solitary CALS. This can lead to the activation of melanocytes which strengthens the synthesis of melanin in the melanosomes that may cause multiple CALSs [810]. As we know, melasma or PIH can be developed due to the increased secretion of Basic Fibroblast Growth Factor (bFGF) from the damaged keratinocyte. However, the expression of bFGF in the keratinocytes of CALS does not rise and bFGF may not provoke CALS [8-10]. According to the case of a giant solitary CALS followed for 20-30 years, NF1 somatic mutations have not 
been foundin melanocytes and keratinocytes in the epidermis and fibroblasts in the dermis [11]. Not only that, the development of a giant solitary CALS into neurofibromatosis was never found [11]. However, if more than 6 multiple CALSs occur even without neurofibromatosis, there is a possibility that NF1 somatic mutations may occur in the epidermal malanocytes over time. In the case of more than 6 multiple CALS, they may appear without neurofibromatosis at first, but eventually develop into neurofibromatosis $[1,7,11]$. The histopathological findings of CALS show moderate elongation of rete ridge with increased number of melanocytes and increased melanin deposition in the epidermis, and a few melanophages in the upper dermis. CALS does not transform into a malignant lesion due to the nonexistence of nerve cell [1,2]. Recently, traditional laser treatments such as ruby laser, alexandrite laser and $532 \mathrm{~nm}$ Potassium Titanyl Phosphate (KTP) laser have been widely used in the CALS treatment. However, traditional laser treatments were somewhat unsatisfactory since they produced purpurae, crusts, PIH, mottled hypopigmentation and scarring [4-6]. In particular, it is extremely difficult to treat infantile CALS without causing PIH [4-6]. There are several possible reasons why traditional laser therapy for CALS may end up with undesirable results.Generally, the $532 \mathrm{~nm}$ wavelength of the KTP laser, the $694 \mathrm{~nm}$ wavelength of the ruby laser, the 515-755 $\mathrm{nm}$ wavelength of intense pulsed lights, and the $755 \mathrm{~nm}$ wavelength of the alexandrite laser are absorbed by melanin much more compared to the $1064 \mathrm{~nm}$ wavelength of QSNL $[12,13]$. This higher absorbance to melanin produces laser energy that not only destroys epidermal melanocytes but also damages surrounding keratinocytes in the lesions at the same time $[12,13]$. The damaged keratinocytes secrete interleukin1(IL-1), which stimulates keratinocytes to secrete some keratinocytic injury-induced cytokines such as endothelin-1, $\alpha$-Melanocyte Stimulating Hormone (MSH), Adrenocorticotropic Hormone (ACTH) and prostaglandin(PGE2, PGF2 $\alpha$ ).These melanogenic cytokines activate melanocytes, which enhance melanin synthesis in melanosomes, thereby causing PIH and aggravating CALS [8-10]. The damaged keratinocytes secrete the single-chain urokinase type plasminogen activator(sc-uPA), which converts plasminogen into plasmin and the plasminstimulated keratinocytes secrete bFGF.Then melanocytes are activated by this bFGF, which result in an increase in melanin synthesis in melanosomes, finally leading to PIH [8-10]. However, if bFGF is not increased in the lesional keratinocytes of CALS, bFGF may not trigger CALS $[12,13]$. In the traditional laser treatments, the laser energy, which is strong enough to lead to purpurae and crusts, causes damage to fibroblasts, mast cells, lymphocytes, macrophages and vascular endotheliums simultaneously.Especially, Stem Cell Growth Factor (SCF) and Hepatocyte Growth Factor (HGF) secreted from damaged fibroblasts activate melanocytes to increase melanin synthesis in melanosomes and eventually induce $\mathrm{PIH}$, therefore exacerbating CALS[8-10]. Finally, the reactive oxygen species such as free radical oxygen and peroxide or nitric oxide generated by damaged keratinocytes activate melanocytes and strengthen melanin synthesis in melanosomes, eventually leading to PIH and exacerbating CALS [8-10]. The authors devised a new treatment using a Dr.Hoon Hur's GPT with a high fluence1064nm QSNL (StarWalkerLaser, Fotona, Slovenia).The parameters were a spot size of $7 \mathrm{~mm}$, a fluence of $2.2 \mathrm{~J} / \mathrm{cm}^{2}$ and a pulse rate of $10 \mathrm{~Hz}$ with one pass by a sliding-stacking technique over the infantile CALS at intervals of one week.We believe that Dr. Hoon Hur's GPT using a high fluence $1064 \mathrm{~nm}$ QSNL is safer and more effective than the traditional CALS treatments [12-15]. In previous papers, the authors also reported that Dr. Hoon Hur's GPT using a high 1064 $\mathrm{nm}$ QSNL is a very effective therapy without harmful side effects such as PIH, mottled hypopigmentation or scarring in various pigmentary skin diseases such as café au lait spot [12-15], partial unilateral lentiginosis [13,16], Becker's nevus [13], Ota's nevus [17], Hori's nevus [18], congenital melanocytic nevus [19], Riehl's melanosis [20], erythema ab igne [21] and prurigo pigmentosa [22]. We think that Dr. Hoon Hur's GPT, which is performed regularly on a weekly basis with minimal damage to the epidermis, promotes gradual destruction of melanocytes in the epidermis or dermis, leading to apoptotic melanocytic cell death program [1215].Due to the poor absorption by epidermal melanin, Dr. Hoon Hur's GPT with the adoption of high fluence 1064-nm QSNL is able to destroy the melanosomes in the epidermal melanocytes with minimal epidermal damage, finally causing epidermal melanocytes to lose functions and turn into ghost cells [12-15]. The more weekly Dr. Hoon Hur's GPT using a high fluence 1064 $\mathrm{nm}$ QSNL is performed, the more epidermal melanocytes are destroyed and the apoptosis of epidermal melanocytes is promoted. Moreover, the dispersed melanosomes and melanins, the end product of destroyed melanocytes, are either eliminated by transepidermal elimination or by dermal melanophages through the lymphatic system [12-15]. The lesional melanocytes are gradually replaced by normal melanocytes, which migrate from the outer root sheath of hair follicles by apoptotic melanocytic cell death program and homeostasis.Finally, CALS can be completely removed without any side effects or recurrences [12-15]. In this study, all 19 patients with infantile CALS received 20-50 treatment sessions of Dr. Hoon Hur's GPT with a high fluence $1064 \mathrm{~nm}$ QSNL at a one-week interval with a spot size of $7 \mathrm{~mm}$, a fluence of $2.2 \mathrm{~J} / \mathrm{cm}^{2}$ and a pulse rate of $10 \mathrm{~Hz}$ with one pass by a sliding-stacking technique. A single pass of a fluence at $2.2 \mathrm{~J} / \mathrm{cm}^{2}$ by a sliding-stacking technique is critical to minimize epidermal damage [12-15]. If double passes of a fluence of $2.2 \mathrm{~J} /$ $\mathrm{cm}^{2}$ by a sliding-stacking technique were performed, the epidermal damages could occur. The damaged keratinocytes might have secreted the melanogenic cytokines such as endothelin-1, $\alpha$-MSH, ACTH, bFGF and prostaglandin (PGE2, $\mathrm{PGF} 2 \alpha$ ), and reactive oxygen species such as free radical oxygen and peroxide or nitric oxide that could induce PIH, eventually exacerbating CALS[12-15]. In short, the end point of Dr. Hoon Hur's GPT with a high fluence $1064 \mathrm{~nm}$ QSNL is not to cause petechiae or purpurae, but to induce erythema only. Since Dr. Hoon Hur's GPT is less absorbed by epidermal melanin, it transmits enough energy without destroying normal background tissue and destroys epidermal melanocytes without crusts and purpurae, eventually preventing PIH and scarring [12-15]. However, in order to remove infantile CALS completely without recurrences, 20-50 continuous treatments is required weekly. In 
this study, we treated all 19 patients with infantile CALS (Figures 1,3,6) using Dr. Hoon Hur's GPT with a high fluence $1064 \mathrm{~nm}$ QSNL.The complete removals of the pigmented lesions were accomplished without PIH and scarring in all 19patients with infantile CALS (Figures 2,4,7). There was no relapse after 6-14 months of follow-up (Figures 5,8). All patients treated with Dr. Hoon Hur's GPT using a high fluence $1064 \mathrm{~nm}$ QSNL were satisfied with the outcome of their therapy.

\section{Conclusion}

In this study, infantile CALS were treated by Dr. Hoon Hur's GPT using a high fluence $1064 \mathrm{~nm}$ QSNL and were completely eliminated without side effects and recurrences.In our view, Dr. Hoon Hur's GPT using a high fluence $1064 \mathrm{~nm}$ QSNL is a new, safe and proper treatment option to achieve complete clearance of infantile CALS.

\section{Referencs}

1. Shah KN. The diagnostic and clinical significance of café-au-lait macules: PediatrClin North Am. 2010; 57(5):1131-1153.

2. Landau M, Krafchik BR. The diagnostic value of café-au-lait macules.J Am AcadDermatol. 1999;40(6 Pt 1):877-890.

3. Cohen JB, Janniger CK, Schwartz RA. Café au lait spots.PaediatrDermatol. 2000;66:22-24

4. Michel S, Hohenleutner U, Baumler W. Dermatological treatment with the Q-switched ruby laser: Indication and application. Hautarzt. 1997;48(7):462-470.

5. Polder KD, Landau JM, Vergilis IJ, Goldberg LH, Friedman PM, Bruce S. Laser eradication of pigmented lesions: a review. Dermatol Surg. 2011;37(5):572-595.

6. Belkin DA, Neckman JP, Jeon H, Paul F, Roy GG. Response to Laser Treatment of Café au Lait Macules Based on Morphologic Features. JAMA Dermatol. 2017;153(11):1158-1161.

7. De Schepper S, Boucneau J, Vander HY, Messiaen L, Naeyaert JM, Lambert J, et al. Café-au-lait spots in neurofibromatosis type 1 and in healthy control individuals: hyperpigmentation of a different kind? Arch Dermatol Res. 2006;297:439-449.

8. Hattori H, Kawashima M, Ichikawa Y, Imokawa G. The epidermal stem cell factor is over-expressed in lentigosenilis: implication for the mechanism of hyperpigmentation. J Invest Dermatol. 2004;122(5):1256-1265.

9. Okazaki M, Yoshimura K, Suzuki Y, Uchida G, Kitano Y, Harii K, et al. The mechanism of epidermal hyperpigmentation in cafe au lait macules of neurofibromatosis type 1 may be associated with dermal fibroblastderived stem cell factor and hepatocyte growth factor. Bri J Dermatol. 2003;148(4):689-697.

10. Okazaki M, Youshimura K, Uchida G, Suzuki Y, Kitano Y, Harii K. Epidermal hyperpigmentation in non-syndromic solitary cafe au lait macules may be associated with increased secreation of endothelin-1 by lesional keratinocytes. J Plastic Sur \& hand Sur. 2005;39(4):213217.

11. Nguyen JT, Yan AC, James WD. Large solitary café au lait spots: a report of 5 cases and review of the literature. Cutis. 2004;73(5):311-316.

12. Hur H. The Treatment of Café Au Lait Spot Using Dr. HoonHur's Golden
Parameter Therapy. J Dermatol Ther. 2016;1(1):1-4.

13. Hur H, Kim YR, Shim DT. The Treatment of Café Au Lait Spot, Partial Unilateral Lentiginosis and Becker's Nevus Using a High Fluence 1064nm Q-swithedNd:YAG Laser. J Clin and Cosmet Dermatol 2017;1(2):1-4.

14. Baek JO, Park IJ, Lee KR, Ryu HR, Kim JS, Lee SK, et al. High-fluence 1064-nm Q-Switched Nd:YAG laser: Safe and effective treatment of café-au-lait macules in Asian patients. J Cosmet Dermatol. 2018;17(3):380-384.

15. Hur H, Kim JH, Park IJ, Park CH, Shim DT, Sung IN, et al. The New Treatment of Café Au Lait Spot Using Dr. HoonHur's Golden Parameter Therapy With a High Fluence 1064nm Q-Switched Nd:YAG Laser. Int J Cur Res. 2018;10(4):68082-668086.

16. Hur H, Choi YJ, Cheon MS, Kim YR. The New Treatment of Partial Unilateral Lentiginosis Using Dr. HoonHur's Golden Parameter Therapy With a High Fluence 1064nm Q-Switched Nd:YAG Laser Without Side Effects. Int J Cur Res. 2017;9(12):63456-63460.

17. Hur H, Park CH, Kim YR, Hyun DN. Treatment of Ota's Nevus Using Dr. HoonHur's Golden Parameter with a High Fluence 1064nm Nd: YAG Laser without Side Effects. J Dermatol Res and Ther. 2017;3(2):1-4.

18. Hur H, Kim YR. The Treatment of Hori's Nevus by New Combination Treatment without Side Effects: Dr. HoonHur's Golden Parameter Therapy and Dr. HoonHur's Optimal Melanocytic Suicide-2 Parameter Therapy. J Clin Res Dermatol. 2017;4(5):1-5.

19. Hur H, Park CH, Kim YR, Kim PS. Treatment of a Congenital Melanocytic Nevus by New Combination Therapy: Intense Pulse Light Therapy and Dr. HoonHur's Golden Parameter Therapy. J DermatolTher. 2017;1(1):12-16.

20. Hur H, Lee GH, Kim PS, Hyun DN, Kim YR. The Treatment of Riehl'sMelanosis Using Dr. HoonHur's Golden Parameter Therapy With a High Fluence 1064nm Q-Switched Nd:YAG Laser Without Side Effects. Int J Cur Res. 2018;10(2):65103-65108.

21. Hur H, In SI, Cheon MS, Choi YJ, Lee DH, Kim YR. The Treatment of Erythema AbIgne Using Dr. HoonHur's Golden Parameter Therapy With a High Fluence 1064nm Q-Switched Nd:YAG Laser. Int J Cur Res. 2018;10(10):74218-74221.

22. Hur H, Shim DT, Cheon MS, Kim PS, Hyun DN, Kim YR. The Treatment of Postinflammatoty Hyperpigmentation Due To PrurigoPigmentosa Using Dr. HoonHur's Golden Parameter Therapy With a High Fluence 1064nm Q-Switched Nd:YAG Laser. Int J Cur Res. 2019;11(3):24912494. 